Of the 61 black patients, one $\mathrm{C} / \mathrm{T}$ heterozygote was found (Fig. 1B) whereas all the 64 black controls were of the wild type. A DNA sequence analysis was subsequently performed and confirmed heterozygosity for the $\mathrm{C} / \mathrm{T}$ polymorphism, $1 \mathrm{bp}$ upstream from the known 20210G/A mutation at position 20209. A review of the medical history of this patient revealed that he was a 79 year-old Jamaican male with prostatic carcinoma and proximal DVT. The C20209T polymorphism was not found in any of the 50 healthy Asian Indians or 69 healthy South East Asians tested.

PT20209C/T is a novel polymorphism previously reported in 4 unrelated African Americans but not in white Americans (4). We have demonstrated a low prevalence $(1.6 \%)$ of this polymorphism in 61 black subjects with venous thrombosis and an absence of the polymorphism in healthy black controls, Asian Indians and SE Asians. The true prevalence of the PT20209C/T polymorphism cannot be defined in a small case-control study but it would appear to be lower in our study population (heterozygosity $0.8 \%$ ) compared to African-Americans (heterozygosity $4.9 \%$ ). Interestingly, in an unpublished study of 503 indi- viduals in the UK (unknown ethnicity) referred for standard thrombophilia tests, only one $\mathrm{C} / \mathrm{T}$ heterozygote was detected. Thus, it would appear that the frequency of this mutation in the UK population is not high. The clinical significance of the 20209C/T polymorphism among the black population remains unclear and there are as yet insufficient data to suggest a possible prothrombotic role. Whether the PT20209C/T polymorphism is associated with elevated prothrombin levels is unknown but this may be a possibility given its proximity to PT20210G/A. Further research is required to clarify the prevalence and significance of the PT20209C/T in the black population.

Peng Yen Soo', Raj K. Patel', Steve Best', Roopen Arya², Swee Lay Thein ${ }^{1,2}$

'Department of Haematological Medicine, Guy's, King's and St Thomas' School of Medicine, King's College London, London, UK 2Department of Haematological Medicine, King's College Hospital, London, UK

\title{
References
}

1. Dowling NF, Austin H, Dilley A, et al. The epidemiology of venous thromboembolism in Caucasians and African-Americans: the GATE Study. J Thromb Haemost 2003; 1: 80-7.
2. Patel RK, Ford E, Thumpston J, et al. Risk factors for venous thrombosis in the black population. Thromb Haemost 2003; 90: 835-8.

3. Rees DC, Chapman NH, Webster MT, et al. Born to clot: the European burden. Br J Haematol 1999; 105: 564-6.
4. Warshawsky I, Hren C, Sercia L, et al. Detection of a Novel Point Mutation of the prothrombin gene at position 20209. Diagn Mol Pathol 2002; 11: 152-6.

5. Patel RK, Ford E, Thumpston J, et al. Coagulation factor levels and venous thrombosis in the black population. Thromb Haemost 2004; 91: 828-30.

\section{A functional serotonin transporter (SLC6A4) polymorphism modifies the association of smoking and diabetes with asymptomatic carotid atherosclerosis}

\section{Dear Sir,}

Serotonin (5-HT), a crucial mediator of platelet activation, is conveyed into the cells through a membrane transporter. There is a 44-bp insertion/deletion polymorphism (long [L] and short [S] allele) in the promoter region of the SLC6A4 gene, coding for the 5-HT transporter. The $\mathrm{L}$ allele is associated with higher promoter activity (1), increased 5-HT uptake in platelets (2) and

\footnotetext{
*Both authors contributed equally.
}

\section{Correspondence to:}

Birger Wolff, MD

Klinik für Innere Medizin B

Ernst-Moritz-Arndt-Universität Greifswald

Friedrich-Loeffler-Straße 23 a

17487 Greifswald, Germany

Tel.: +49-3834-866656, Fax: +49-3834-866657

E-mail: bwolff@pop.uni-greifswald.de

Received April 26, 2004

Accepted after resubmission October 28, 2004

Financial support:

This work was supported by the German Federal Ministry of Education, Science, Research, and Technology (grant 0 I GG 9845/5, 0IZZ96030) and from the Ministry for

Education, Research and Cultural Affairs and the Ministry for Social Affairs of the State Mecklenburg-Western Pomerania.

Thromb Haemost 2005; 93: I 80-2 with higher 5-HT plasma levels (3). Recently, three case-control studies described an association of the SLC6A4 polymorphism with coronary heart disease (CHD) and myocardial infarction (4-6). Carotid atherosclerosis (CA) measured by ultrasound correlates with existing CHD and is predictive of cardiac events in individuals without clinically evident disease (7). We consequently investigated the association of the SLC6A4 polymorphism with asymptomatic CA among participants of the Study of Health in Pomerania (SHIP).

For this purpose, a total of 629 subjects aged 45 to 79 years had completed genotyping by PCR and ultrasound investigation of the carotid arteries as previously described (8). CA was defined as presence of atherosclerotic plaques in the extracranial carotids on either side (common, internal, external carotids and carotid bifurcation). Hypertension was defined as a systolic blood pressure (BP) of $\geq 160 \mathrm{mmHg}$, a diastolic BP of $\geq 95$ $\mathrm{mmHg}$, or intake of antihypertensives. Smoking was defined as current smoking of at least one cigarette per day (mean daily consumption: $13.4 \pm 8.8$ cigarettes), diabetes as self-reported physician diagnosis of diabetes, or serum haemoglobin $\mathrm{A}_{1 \mathrm{C}}\left(\mathrm{HbA}_{1 \mathrm{C}}\right)$ of $>7.0 \%$. Non-fasting blood samples were taken and lipids and $\mathrm{HbA}_{1 \mathrm{C}}$ were determined according to standard procedures. Data were analyzed using ANOVA and logistic regression. Gene-to- 
Table I: ((author: add legend))Odds ratios and confidence intervals were calculated by backward and forward stepwise regression analyses with $C A$ as dependent variable and as independent variables: age (I0-year increments), sex, diabetes, hypertension, smoking, BMI, total cholesterol/HDL ratio and genotype as independent variables. ${ }^{*} p<0.05$, $\dagger p<0.01, \neq p<0.001$

\begin{tabular}{|c|c|c|c|}
\hline & Subjects (\%) & Odds ratio & $95 \% \mathrm{Cl}$ \\
\hline $\begin{array}{l}\text { Age, per 10-year in- } \\
\text { crement }\end{array}$ & - & 2.74 & $2.10-3.59 \ddagger$ \\
\hline Male sex, $y / n$ & $186(29.6)$ & 1.69 & $1.05-2.70^{*}$ \\
\hline Hypertension, $y / n$ & $375(59.6)$ & 1.87 & $1.22-2.87^{\dagger}$ \\
\hline Smoking, $y / n$ & $95(15.1)$ & 1.37 & $0.78-2.36$ \\
\hline BMI, per I-SD & - & 1.37 & $1.03-1.70^{*}$ \\
\hline Diabetes, $y / n$ & $97(15.4)$ & 1.27 & $0.66-2.46$ \\
\hline $\begin{array}{l}\text { Total Cholesterol/ } \\
\text { HDL ratio, per I-SD }\end{array}$ & - & 1.36 & $0.97-1.62$ \\
\hline LS genotype, $y / n$ & $290(46.1)$ & 1.06 & $0.62-1.83$ \\
\hline LL genotype, $y / n$ & $235(37.4)$ & 1.17 & $0.66-2.07$ \\
\hline L allele, y/n & 525 & 1.11 & $0.66-1.85$ \\
\hline $\begin{array}{l}\text { LS genotype and } \\
\text { smoking }\end{array}$ & $38(6.0)$ & 6.70 & $1.31-34.37 *$ \\
\hline $\begin{array}{l}\text { LL genotype and } \\
\text { smoking }\end{array}$ & $44(7.0)$ & 8.97 & $1.76-45.83^{\dagger}$ \\
\hline L allele and smoking & $82(13.0)$ & 7.85 & $1.25-28.65^{\dagger}$ \\
\hline $\begin{array}{l}\text { LS genotype and dia- } \\
\text { betes }\end{array}$ & $47(7.5)$ & 5.95 & $1.07-33.08 *$ \\
\hline $\begin{array}{l}\text { LL genotype and dia- } \\
\text { betes }\end{array}$ & $35(5.6)$ & 5.86 & $0.98-35.45$ \\
\hline $\mathrm{L}$ allele and diabetes & $82(13.0)$ & 5.98 & $1.25-28.65^{*}$ \\
\hline
\end{tabular}

environment interactions between genotype and cardiovascular risk factors were tested by backward and forward stepwise regression analyses.

\section{Results}

The percentages of the SLC6A4 LL, LS, and SS genotypes in the sample were $37.4 \%, 46.1 \%$, and $16.5 \%$. As shown in the table, age, sex, hypertension, and BMI, yet not SLC6A4 genotype, were independently related to the prevalence of CA. However, $\mathrm{L}$ allele carriership was associated with greatly increased odds ratios for CA among smokers and also among diabetics.

\section{Discussion}

In this study, we observed that, among smokers and diabetics, the SLC6A4 L allele is associated with an increased prevalence of $\mathrm{CA}$. Although it has naturally to be emphasized that these interactions are effective for the definitions of smoking (i.e. only current smoking) and diabetes (previously established diagnosis of diabetes and/or $\mathrm{HbA}_{1 \mathrm{C}}$ of $>7.0 \%$ ) as specifically applied in this study.

To date, the SLC6A4 polymorphism has been related to CHD and myocardial infarction in three case-control studies (5-7). In one of them, it was shown that the association between the L allele and CHD was particularly pronounced among smokers (6). However, no such interaction could be shown for diabetics. In another investigation (7), individuals with the SS genotype were protected from early-onset myocardial infarction, especially those who were smokers. Protective properties of the SS genotype among diabetics, though, were likewise not found in this study. Thus, although no direct association between genotype and CA was established, our results add support to the findings from these studies and to the hypothesis that the 5-HT transporter polymorphism is related to cardiovascular disease. It seems important to note that both smoking and diabetes are conditions associated with increased platelet activation. Smoking augments platelet-dependent thrombin generation and thrombus formation (10), platelets from smokers are refractory to the antiaggregating impact of nitroglycerin (11) and they exhibit a greater $5-\mathrm{HT}_{2 \mathrm{~A}}$ - and GPIIb/IIIa receptor density (12). Similarly, platelets from diabetics show an enhanced response upon stimulation with collagen and thrombin (13) which is to some extent mediated through 5-HT (14).

In summary, we have shown in a non-clinical epidemiological cohort that the L-allele of the SLC6A4 polymorphism is associated with a 7.85-fold risk in smokers and a 6-fold risk in diabetics of CA. This association is mediated through an interaction with smoking and diabetes and may be attributable to a modification of 5-HT dependent platelet activation.

\section{Birger Wolff'*, Hans J. Grabe ${ }^{2 *}$, Henry Völzke ${ }^{3}$, Jan Lüdemann ${ }^{3}$, Christian Schwahn ${ }^{4}$, Harald J. Freyberger ${ }^{2}$, Ulrich John ${ }^{3}$, Martina Lange $^{5}$, Ingolf Cascorbi ${ }^{5}$, Stephan B. Felix \\ 'Klinik für Innere Medizin B, ${ }^{2}$ Klinik für Psychiatrie und Psycho- therapie, ${ }^{3}$ Institut für Epidemiologie und Sozialmedizin, ${ }^{4}$ Klinik für Zahnheilkunde, ${ }^{5}$ Institut für Pharmakologie, Peter Holtz Research Center of Pharmacology and Experimental Therapeutics, Universi- tät Greifswald, Greifswald, Germany}

\section{References}

1. Lesch KP, Bengel D, Heils A, et al. Association of anxiety-related traits with a polymorphism in the serotonin transporter gene regulatory region. Science 1996; 274: $1527-31$

2. Greenberg BD, Tolliver TJ, Huang SH, et al. Genetic variation in the serotonin transporter promoter region affects serotonin uptake in human blood platelets. Am J Med Genet 1999; 88: 83-7.

3. Hanna GL, Himle JA, Curtis GC, et al. Serotonin transporter and seasonal variation in blood serotonin in families with obsessive-compulsive disorder. Neuropsychopharmacology 1998; 18: 102-11.
4. Fumeron F, Betoulle D, Nicaud V, et al. Serotonin transporter gene polymorphism and myocardial infarction: Etude Cas-Temoins de l'Infarctus du Myocarde (ECTIM). Circulation 2002; 105: 2943-5.

5. Arinami T, Ohtsuki T, Yamakawa-Kobayashi K, et al. A synergistic effect of serotonin transporter gene polymorphism and smoking in association with CHD. Thromb Haemost 1999; 81: 853-6.

6. Coto E, Reguero JR, Alvarez V, et al. 5-Hydroxytryptamine 5-HT2A receptor and 5-hydroxytryptamine transporter polymorphisms in acute myocardial infarction. Clin Sci 2003; 104: 241-5.
7. Craven TE, Ryu JE, Espeland MA, et al. Evaluation of the associations between carotid artery atherosclerosis and coronary artery stenosis. Circulation 1990; 82 : $1230-42$.

8. Wolff B, Volzke H, Ludemann J, et al. Association between high serum ferritin levels and carotid atherosclerosis in the Study of Health in Pomerania (SHIP). Stroke 2004; 35: 435-57.

9. Hioki H, Aoki N, Kawano K, et al. Acute effects of cigarette smoking on platelet-dependent thrombin generation. Eur Heart J 2001; 22: 56-61. 
10. Haramaki N, Ikeda H, Takajo $Y$, et al. Long-term smoking causes nitroglycerin resistance in platelets by depletion of intraplatelet glutathione. Arterioscler Thromb Vasc Biol 2001; 21: 1852-6.

11. Markovitz JH, Tolbert L, Winders SE. Increased serotonin receptor density and platelet GPIIb/IIIa acti- vation among smokers. Arterioscler Thromb Vasc Biol 1999; 19: 762-6.

12. Li Y, Woo V, Bose R. Platelet hyperactivity and abnormal calcium homeostasis in diabetes mellitus. Am J Physiol Heart Circ Physiol 2001; 280: 1480-9.
13. Pietraszek MH, Takada Y, Takada A, et al. Blood serotonergic mechanisms in type 2 (non-insulin-dependent) diabetes mellitus. Thromb Res 1992; 66: 765-74.

\section{Inverse correlation between phenylacetate hydrolase activity of the serum PONI pro- tein and homocysteinemia in humans}

\section{Dear Sir,}

Hyperhomocysteinemia is an independent risk factor for cardiovascular disease and stroke, while also being a risk factor for neurodegenerative and renal disorders. However, the actual pathological mechanisms of increased serum levels of Homocysteine (Hcy) or its metabolites in human remain elusive.

The determinants of plasma Hcy concentration are both genetic as well as environmental. Moderate Hcy elevations can be due to homozygosity of the $677 \mathrm{~T}$ allele of the MTHFR gene or nutritional deficiencies in the cofactors (vitamin B6 or B12) or substrate (folate) of the enzymes involved in Hcy metabolism. Severe hyperhomocysteinemia is often caused by inborn error of metabolism such as in Cystathionine Beta Synthase (CBS) deficiency.

Hcy is synthesized during the conversion of dietary methionine to cysteine. Once synthesized, Hcy can be recycled to me-

\section{Correspondence to:}

Jean François Chassé

INSERM UMR S-490

Université Paris V

45 rue des saints Pères

Paris, Cedex 6

75270 France

Tel.: 33 | 42862073, Fax: 33 | 42862072

E-mail: jean-francois.chasse@univ-paris5.fr

Received July 30, 2004

Accepted after revision September 9, 2004

Thromb Haemost 2005; 93: I82-3 thionine by the remethylation pathway or converted into cystathionine by the transulfuration pathway. Hcy is also metabolically converted to form homocysteine thiolactone (HTL) by a metabolic error-editing process in which Hcy is inactivated by methionyl-tRNA synthethase and is released as free HTL. HTL has been implicated in the pathology of hyperhomocysteinemia probably through increased protein homocysteinylation (1).

Paraoxonase 1 (PON1) is secreted by the liver and is a protein component of the high-density lipoproteins. Paraoxonase displays esterase activity and can hydrolyse various exogenous and endogenous substrates (2). It degrades oxidized lipids and is thus considered as a protective anti-oxidant enzyme. Several studies have shown increased susceptibility to cardiovascular disease in individuals carrying polymorphisms in the PON1 gene (3). Interestingly, paraoxonase has been recently shown to degrade HTL (4) and thus could contribute to the detoxification of this metabolite of Hcy. Moreover, in the murine model of severe hyperhomocysteinemia due to CBS deficiency, the activity of PON1 in the liver was downregulated 3-fold (5). The moderate to severe hyperhomocysteinemia in mice, caused by a hyperhomocysteinemic diet or by a genetic deficiency in CBS, is associated with reduced liver PON1 activity, with a downregulation of PON1 mRNA in the liver (6). We therefore hypothesized that homocysteine serum levels could influence serum paraoxonase activity in humans.

Blood samples from 112 subjects with various homocysteinemia were recruited from the Hospital Europeen Georges Pompidou (HEGP-France) in the different clinical units of hos-
Figure I: Relation between homocysteine concentration and relative phenylacetate hydrolase activity.

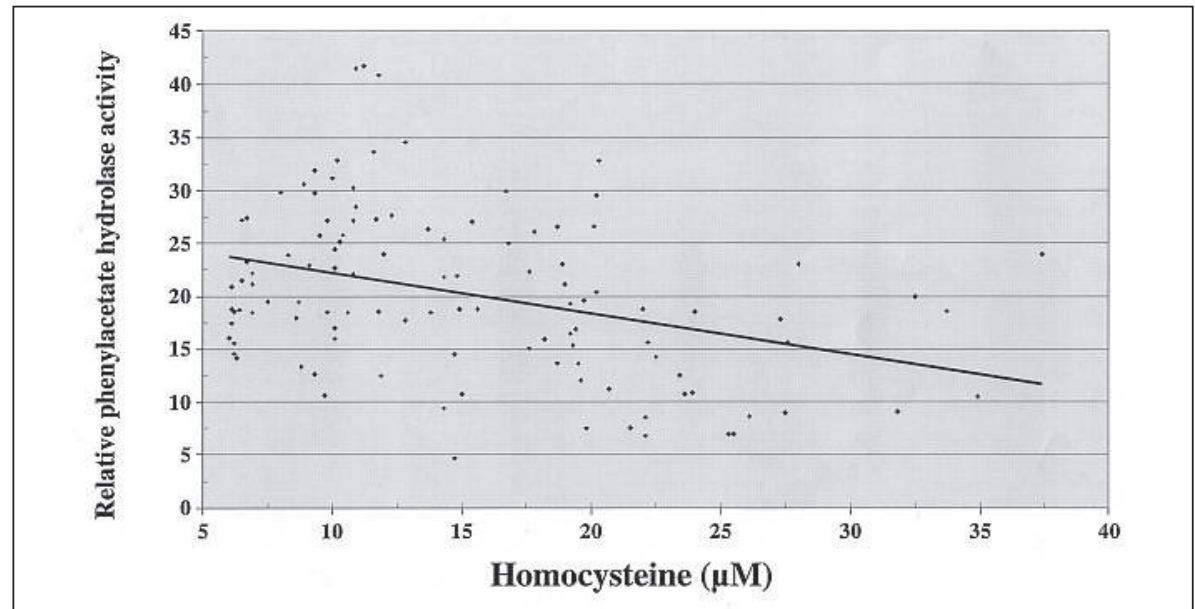

